MATHEMATICS OF COMPUTATION

Volume 71, Number 237, Pages 311-318

S 0025-5718(01)01333-3

Article electronically published on July 22, 2001

\title{
AUTOMATIC REDUCTION OF ELLIPTIC INTEGRALS USING CARLSON'S RELATIONS
}

\author{
NORMAN GRAY
}

\begin{abstract}
In a series of papers, B. C. Carlson produced tables of elliptic integrals, evaluating them in terms of easily computed symmetrical functions, using a group of multivariate recurrence relations. These relations are, however, cumbersome to use by hand and, in the absence of a specific reductive algorithm, difficult to use with computer algebra. This paper presents such an algorithm, guaranteed to reduce a general elliptic integral to a set of fundamental ones.
\end{abstract}

\section{InTRODUCTION}

An elliptic integral is an integral of the form

$$
\int R(t, s(t)) \mathrm{d} t
$$

where $s^{2}$ is a third- or fourth-order polynomial in $t$, with simple zeros, and $R(t, s)$ is a rational function of its arguments, containing at least one odd power of $s$. The evaluation and reduction of such integrals has a long history, reviewed in Car98.

In a series of papers, Carlson discussed such integrals Car87 Car88, Car89. Car91, written in the form

$$
[p] \equiv\left[p_{1}, p_{2}, \ldots, p_{N}\right] \equiv \int_{y}^{x}\left(a_{1}+b_{1} t\right)^{p_{1} / 2} \cdots\left(a_{N}+b_{N} t\right)^{p_{N} / 2} \mathrm{~d} t
$$

where $p_{1}, \ldots, p_{N}$ are nonzero integers, the integrand is real, and the integral is assumed to be well defined. If three or four of the $p_{i}$ are odd, then this integral is elliptic, and may be reduced either to the usual Legendre forms, or to the more symmetric forms

$$
\begin{aligned}
R_{F}(x, y, z) & =\frac{1}{2} \int_{0}^{\infty}[(t+x)(t+y)(t+z)]^{-1 / 2} \mathrm{~d} t \\
R_{J}(x, y, z, \rho) & =\frac{3}{2} \int_{0}^{\infty}[(t+x)(t+y)(t+z)]^{-1 / 2}(t+\rho)^{-1} \mathrm{~d} t \\
R_{C}(x, y) & =R_{F}(x, y, y) \\
R_{D}(x, y, z) & =R_{J}(x, y, z, z) .
\end{aligned}
$$

The reduction to Legendre form is usually done using tables such as [GR94]. Instead of this, reduction to Carlson's integrals has the advantages described in [Car87]: the

Received by the editor August 16, 1999 and, in revised form, May 5, 2000.

2000 Mathematics Subject Classification. Primary 33E05; Secondary 33-04.

Key words and phrases. Carlson elliptic integrals. 
interval of integration need not begin or end at a branch point of the integrand, and the symmetry of Carlson's integrals eliminates many cases. As well, the integrals above can be numerically evaluated very efficiently.

If a given integral is not in Carlson's tables, it can be reduced to one that is, or reduced completely to the fundamental integrals, using recurrence relations. Unlike the reduction to Legendre form, this reduction is analytically simple but algebraically overpowering, especially in the case of higher values of $p_{i}$ : this is characteristic of a problem which could be usefully attacked using computer algebra, as long as there is some algorithm to automatically determine which sequence of relations is to be used. I present such an algorithm, which is guaranteed to converge to a sum of fundamental integrals. These integrals are still in the form in (1). In a large number of important cases, however $(N \leq 4$, at most two factors complex (Car91]), these integrals may be reduced analytically, as mentioned above (2). It is the aim of this present work to reduce to as small a size as possible the set of irreducible integrals requiring further analytic or numerical work.

There have been previous schemes for performing such reductions automatically. NP76] describes several such schemes, and the very serious problems from which they suffer, such as a need for expert intervention or intractable intermediate expression swell. In [Car99] (and summarised in [Car98]), Carlson describes an algorithm which will reduce elliptic integrals in stages, first performing an explicit partialfraction decomposition to reduce a general elliptic integral to a sum of simpler ones, then using a one-parameter recurrence relation to reduce these to basic ones, and finally translating these to a small set of fundamental ones which can be evaluated efficiently (namely the $R_{J}, R_{F}, R_{D}$ and $R_{C}$ described above). This is an algorithm which reduces the integrals in a small number of elaborate steps, which is different from the algorithm in the present paper, which uses a potentially large number of simple steps, although the simple expedient of caching intermediate results dramatically reduces the number of steps required (see Section 4). The present algorithm uses only the multivariate recurrence relations described in Car87, Car88, which means it has the same domain of validity as those relations.

\section{The ALGORITHM}

2.1. Notation. Throughout the following, I assume that the integrals have $m \geq 1$ odd powers, and $n \geq 0$ even ones, where $m+n=N$. I do not assume that $m$ is only 3 or 4 , or that the $a_{i}$ and $b_{i}$ are real.

We assume $x>y$ and $a_{i}+b_{i} t>0, y<t<x$, for $i=1, \ldots, N$. Define

$$
d_{i j} \equiv a_{i} b_{j}-a_{j} b_{i}
$$

and

$$
X_{i} \equiv\left(a_{i}+b_{i} x\right)^{1 / 2}, \quad Y_{i} \equiv\left(a_{i}+b_{i} y\right)^{1 / 2} .
$$

We may then define

$$
A\left(p_{1}, \ldots, p_{N}\right) \equiv X_{1}^{p_{1}} \cdots X_{N}^{p_{N}}-Y_{1}^{p_{1}} \cdots Y_{N}^{p_{N}} .
$$

If one limit of integration is a branch point of the integrand, then $X_{i}$ or $Y_{i}$ is zero for some value of $i \leq m$. If both limits of integration are branch points, the elliptic integral is called complete.

This notation is defined in [Car88, but unlike that paper, I shall assume, for the sake of simplicity, that $b_{i} \neq 0$; an integral with $m=4$, say, and one $b_{i}=0$, is really 
an integral with $m=3$. I shall also assume that $d_{i j} \neq 0$; since the relation $d_{i j}=0$ is equivalent to proportionality of $a_{i}+b_{i} t$ and $a_{j}+b_{j} t$, an integral with $d_{i j}=0$ should be taken to be an integral with one lower $m$.

Carlson provides four recurrence relations to aid in the reduction to fundamental integrals. These are as follows.

Let $e_{i}$ be an $N$-tuple with 1 in the $i$ th place and zeros elsewhere, so that $\left[p+2 e_{1}\right]=$ $\left[p_{1}+2, p_{2}, \ldots, p_{N}\right]$.

$$
\left(p_{1}+\cdots+p_{N}+2\right) b_{i}[p]=\sum_{j \neq i} p_{j} d_{j i}\left[p-2 e_{j}\right]+2 A\left(p+2 e_{i}\right) .
$$

The next two relations are equivalent to each other:

$$
\begin{aligned}
d_{i j}[p] & =b_{j}\left[p+2 e_{i}\right]-b_{i}\left[p+2 e_{j}\right], \\
b_{j}[p] & =b_{i}\left[p-2 e_{i}+2 e_{j}\right]+d_{i j}\left[p-2 e_{i}\right] .
\end{aligned}
$$

Finally

$$
d_{i j}[p]=d_{k j}\left[p+2 e_{i}-2 e_{k}\right]+d_{i k}\left[p+2 e_{j}-2 e_{k}\right] .
$$

To these we add two further derived ones. The first is a rearrangement of $(\mathrm{A} i)$ :

$$
\begin{gathered}
\left(p_{j}+2\right) d_{j i}[p]=\left(p_{1}+\cdots+p_{N}+4\right) b_{i}\left[p+2 e_{j}\right]-2 A\left(p+2 e_{i}+2 e_{j}\right) \\
-\sum_{k \neq i, j} p_{k} d_{k i}\left[p+2 e_{j}-2 e_{k}\right] .
\end{gathered}
$$

The second is obtained by using $(\mathrm{C} j k)$ on all but one of the terms in the sum in $(\mathrm{A} i)$ :

$$
\begin{aligned}
(\mathrm{AC} i j) \quad\left(p_{1}+\cdots+p_{N}+2\right) b_{i}[p] & =p_{j} d_{j i}\left[p-2 e_{j}\right]+2 A\left(p+2 e_{i}\right) \\
& +\sum_{k \neq i, j} \frac{p_{k} d_{k i}}{b_{k}}\left(b_{j}\left[p-2 e_{j}\right]+d_{j k}\left[p-2 e_{k}-2 e_{j}\right]\right) .
\end{aligned}
$$

Note that all of these relations involve the powers of $p_{i}$ being raised or lowered in increments of two.

Carlson defined four fundamental integrals in the quartic case (see Section 2.2), and three further ones in the cubic case. We modify this set, for both notational convenience and generality, to the case where there are $m$ odd, and $n$ even powers. Define

$$
\begin{aligned}
{ }_{m n} C_{0} & \equiv[\underbrace{+1,+1, \ldots,+1}_{m}, \underbrace{0, \ldots, 0}_{n}], \\
{ }_{m n} C_{1} & \equiv[-1,+1, \ldots,+1,0, \ldots, 0], \\
\vdots & \\
{ }_{m n} C_{N} & \equiv[+1,+1, \ldots,+1,0, \ldots, 0,-2],
\end{aligned}
$$

and, in general, ${ }_{m n} C_{i}(1 \leq i \leq N)$ is ${ }_{m n} C_{0}$ with $\left(p_{i} \rightarrow p_{i}-2\right)$. Since trailing zero powers make no difference, all the integrals ${ }_{m n} C_{i}$, with $n>i-m$, are equivalent.

Define a target vector

$$
{ }_{m n} \tau \equiv(\underbrace{+\frac{1}{2}, \ldots,+\frac{1}{2}}_{m}, \underbrace{-\frac{1}{2}, \ldots,-\frac{1}{2}}_{n})
$$


where each component of ${ }_{m n} \tau$ is one-half less than the corresponding component of ${ }_{m n} C_{0}$ (it could be any amount strictly between zero and one), and define $\epsilon_{i} \equiv$ $p_{i}-\tau_{i}(1 \leq i \leq N)$. Call a power $p_{i}$ "high" if $\epsilon_{i}>1$ and "low" if $\epsilon_{i}<-1$; adding two to a low $p_{i}$, and subtracting two from a high $p_{i}$, will both reduce $\left|\epsilon_{i}\right|$. Thus, for example, $p_{1}=-1$ is low, but $p_{1}=+1$ is neither high nor low (there is no significance to the definitions of high and low relying on strict inequalities: since $p_{1}$ to $p_{m}$ are odd and $p_{m+1}$ to $p_{N}$ are even integers by hypothesis, $\epsilon_{i}$ can never be integer and, a fortiori, never equal to either -1 or +1$)$. Define $\sigma_{N} \equiv \sum_{i=1}^{N}\left|\epsilon_{i}\right|$. With these definitions, term a recurrence relation "applicable" if, when it is applied, it produces integrals which are closer to the fundamental integrals in (6), in the sense that the parameter $\sigma_{N}$ for each of the resultant integrals is strictly less (by a finite amount) than that for the original integral.

By enlarging the set of recurrence relations above, we have obtained a set which is such that any integral $[p]$ is either one of the fundamental integrals ${ }_{m n} C_{i}$, or has at least one relation applicable. The algorithm consists of identifying an applicable relation for all possible values of $p$. Using this algorithm, every integral $[p]$ may be mechanically reduced towards zero difference $\sigma_{N}$. Since $\sigma_{N}$ decreases monotonically, and the step is finite, and $\sigma_{N}>0$ by construction, the procedure must terminate; at that point, there will be no applicable relations, and all the remaining integrals will be fundamental ones.

2.2. Alternative target: reduction to Carlson's integrals. The fundamental integrals defined above are not the same as those defined by Carlson. At the expense of some symmetry, we may define

$$
\begin{aligned}
{ }_{m n} C_{0}^{\prime} & \equiv[+1,-1, \ldots,-1,0, \ldots, 0], \\
{ }_{m n} \tau^{\prime} & \equiv(\underbrace{+\frac{1}{2},-\frac{3}{2}, \ldots,-\frac{3}{2}}_{m}, \underbrace{-\frac{1}{2}, \ldots,-\frac{1}{2}}_{n}),
\end{aligned}
$$

with corresponding definitions for ${ }_{m n} C_{i}^{\prime}$. Thus the fundamental integrals in Car88] can be written as

$$
I_{3}^{\prime}={ }_{40} C_{0}^{\prime}, \quad I_{3}={ }_{41} C_{5}^{\prime}, \quad I_{1}={ }_{40} C_{1}^{\prime}, \quad I_{2}={ }_{40} C_{4}^{\prime} ;
$$

and the integrals in Car89 are

$$
I_{1 c}={ }_{30} C_{1}^{\prime}, \quad I_{2 c}={ }_{30} C_{0}^{\prime}, \quad I_{3 c}={ }_{31} C_{4}^{\prime},
$$

where all of these integrals can be written in terms of the integrals of (2). There is no real extension beyond Carlson's set here, since, for example, ${ }_{40} C_{3}^{\prime}$ could be transformed either directly into $I_{2}={ }_{40} C_{4}^{\prime}$ by a suitable permutation of the $N$ tuples $a, b$ and $p$, or into a sum of Carlson's integrals through manual use of the recurrence relations.

One does not have complete freedom to choose fundamental integrals and target; see the discussion below the table in subsection 2.3

2.3. Reducing integrals. For each $[p]$ calculate $\epsilon(p)$, and count the number of high and low coefficients, according to the above definitions.

Define $s(\epsilon) \equiv\left\{s_{i}: \epsilon_{s_{1}} \geq \epsilon_{s_{2}} \geq \cdots \geq \epsilon_{s_{N}}\right\}$. If two or more of the $\epsilon_{i}$ are equal, there will be more than one set $\left\{s_{i}\right\}$ which satisfies this condition; these are, however, equivalent, and we may select one set arbitrarily.

For each of the integrals which is not a fundamental one, select the first case in the table which matches the pattern of high and low powers, and apply the 
indicated recurrence relation. Blank entries indicate that there is no corresponding constraint.

$\begin{array}{rccll}\text { case } & \text { high } & \text { low } & \text { constraint } & \text { use } \\ 1 & \geq N-1 & & \sum^{N} p_{i} \neq-2 & \left(\mathrm{~A} s_{N}\right) \\ 2 \mathrm{a} & \geq N-2 & 1 & p_{s_{N}} \neq-2 & \left(\mathrm{~A}^{\prime} s_{N-1} s_{N}\right) \\ 2 \mathrm{~b} & & 1 & p_{s_{N}} \neq-2, \epsilon_{s_{N}}<-2 & \left(\mathrm{~A}^{\prime} s_{N-1} s_{N}\right) \\ 3 & \geq 1 & \geq 2 & & \left(\mathrm{D} s_{N-1} s_{N} s_{1}\right) \\ 4 & \geq 1 & \geq 1 & & \left(\mathrm{C} s_{1} s_{N}\right) \\ 5 & & \geq 2 & & \left(\mathrm{~B} s_{N-1} s_{N}\right) \\ 6 & \geq 1 & 0 & \sum^{N} p_{i} \neq-2 & \left(\mathrm{AC} s_{N} s_{1}\right)\end{array}$

Case 5 will be the first match only in the rather trivial case when $m=2$. There is no match for the cases where there is only one low index $s_{N}$, which is such that $0>\epsilon_{s_{N}} \geq-2$ or $p_{s_{N}}=-2$; however such an integral is deemed fundamental by the definition above (it will be the integral ${ }_{m n} C_{s_{N}}$ ).

The above is valid for a range of choices of fundamental integral ${ }_{m n} C_{i}$ and corresponding $m n \tau$. However, one does not have complete freedom to choose fundamental integrals and targets. For certain choices, it would be possible to construct cases where none of the above cases matched. For example, $p=[3,3,3,-11]$ does not match cases 1 or 6 ; if the target were chosen sufficiently low that this had no low indexes, it would match no cases at all. Similarly, $p=[1,1,-1]$ does not match any case, for the target defined in (7) (since $\epsilon_{3}=-\frac{3}{2}>-2$ ), but this is a fundamental integral by the definition of ${ }_{m n} C_{i}$ above. Finally, $p=[1,-1,-1,-1]$ does not match any case for the target ${ }_{40} \tau^{\prime}$ defined in (9), and this case is unproblematic only because it does match the corresponding fundamental integral ${ }_{40} C_{0}^{\prime}$.

In each of the expressions, the relation would fail if the coefficient on the lefthand side were zero. As noted at the beginning of subsection 2.1 the coefficients $d_{i j}$ and $b_{i}$ are assumed nonzero. In $(\mathrm{A} i),\left(\mathrm{A}^{\prime} i j\right)$ and $(\mathrm{AC} i j)$ the coefficient depends on the set $p_{i}$, and this is reflected in the extra conditions on the $p_{i}$ in those cases.

2.4. Evaluation of the function $A(p)$. In the case where either endpoint of the integration is a branch point, then some $X_{i}$ or $Y_{i}$ will be zero, and $A(p)$ will be undefined if the corresponding $p_{i} \leq 0$.

To help deal with such undefined functions, and also to minimise the number of $A$ functions we must deal with, we may use the two further recurrence relations:

$$
\begin{aligned}
d_{i j} A(p) & =b_{j} A\left(p+2 e_{i}\right)-b_{i} A\left(p+2 e_{j}\right), \\
b_{i} A(p) & =d_{j i} A\left(p-2 e_{j}\right)+b_{j} A\left(p-2 e_{j}+2 e_{i}\right) .
\end{aligned}
$$

The first is (4.8) of [Car88], and the second is obtained by replacing $p$ by $p-2 e_{i}$ and relabelling.

To use these, define a target ${ }_{N} \tau_{A}=\left(\frac{3}{2}, \ldots, \frac{3}{2}\right)$, and take any function $A$ to be fundamental if it has at most one low index. We may add two further cases:

$$
\begin{array}{rlll}
\text { case } & \text { high } & \text { low } & \text { use } \\
7 & \geq 1 & \geq 1 & \left(\mathrm{AF}^{\prime} s_{N} s_{1}\right) \\
8 & & \geq 2 & \left(\mathrm{AF}_{N-1} s_{N}\right)
\end{array}
$$


This procedure will not remove undefined $A(p)$, but will instead transform them into a standard form, in which they either will cancel, or can be dealt with individually.

\section{EXAMPLE}

To illustrate the algorithm in action, I list the sequence of recurrence relations for the integral

$$
I=\int_{0.5}^{2} \sqrt{\frac{(0.3+0.3 t)(0.5+0.1 t)}{0.7-0.1 t}} \frac{\mathrm{d} t}{(0.9-0.3 t)^{2}}
$$

(compare Car99, (6.16)]). This has $m=3, n=1, a=[0.3,0.5,0.7,0.9], b=$ $[0.3,0.1,-0.1,-0.3]$ and $p=[1,1,-1,-4]$. In the first step, with target ${ }_{31} \tau$ as in (7), we find $\epsilon=[1 / 2,1 / 2,-3 / 2,-7 / 2], s=[1,2,3,4]$ and $\sigma_{4}=6$; this $\epsilon$ has no indexes high, and two low, and so matches case 5 , (B34), which reduces the integral to a sum of $p=[1,1,1,-4]$ and $p=[1,1,-1,-2]$. The sequence continues through $\left(\mathrm{A}^{\prime} 34\right),\left(\mathrm{AF}^{\prime} 43\right),(\mathrm{B} 14),(\mathrm{B} 24)$ and (B34) to finish with

$$
\begin{array}{rl}
I=-2.083 \dot{3}_{30} C_{1}-0 & .52083 \dot{3}_{30} C_{2}+0.694 \dot{4}_{30} C_{3}-2.60416 \dot{6}_{31} C_{4} \\
+ & 6.94 \dot{4} A(1,1,1)+8.3 \dot{3} A(1,1,1,-2)=6.24309544
\end{array}
$$

(where the dot accent indicates a repeated decimal). Choosing instead the target ${ }_{31} \tau^{\prime}$, as in subsection 2.2 we find $\epsilon=[1 / 2,5 / 2,1 / 2,-7 / 2], s=[2,1,3,4]$, $\sigma_{4}=7$; this has one high and one low index, and matches case $2 \mathrm{a},\left(\mathrm{A}^{\prime} 34\right)$, reducing the integral to a sum of $p=[1,1,-1,-2], p=[-1,1,-1,-2], p=[1,-1,-1,-2]$ and $A(1,1,1,-2)$. The sequence continues with (C24) and (D142), to finish with

$$
I=-0.3 \dot{3}_{30} C_{1}^{\prime}-0.138 \dot{8}_{30} C_{0}^{\prime}-0.83 \dot{3}_{31} C_{4}^{\prime}+8.3 \dot{3} A(1,1,1,-2)=6.24309544 .
$$

\section{NUMERICAL CHECKS AND EFFICIENCY CONSIDERATIONS}

The algorithm has been checked by implementing it in software, and then automatically reducing all of the distinct integrals with indexes within four of ${ }_{32} C_{0}$, ${ }_{41} C_{0}$, and ${ }_{51} C_{0}$, and evaluating the results numerically. These expressions match to high accuracy.

As is typical of recursive algorithms such as this, there is a danger of the number of intermediate terms becoming unmanageable. To illustrate the extent to which this is a problem, and the degree to which it is solvable, I summarise, in Figure 1 . the results of automatically reducing integrals with $m=3$ and $n \leq 2$, and values of $\sigma$ ranging from $\sigma_{3}=\frac{3}{2}(p=[1,1,1])$ to $\sigma_{5}=22.5(p=[5,5,5,4,4])$. For each distinct value of $\sigma$ which appeared, the figure shows the maximum number of times the algorithm had to invoke one or other recurrence relation, in order to completely reduce the initial integral to fundamental ones. As can be seen, this maximum has a roughly exponential dependence on an integral's initial distance from the target. However, the algorithm is such that it benefits massively from an implementation which caches results, so that no intermediate integral has to be reduced twice. This produces the second curve in Figure [1, in the case of $p=[5,5,5,4,4]$, the number of relations invoked falls from 746046 to just 299, an improvement of over three orders of magnitude. If we improve the caching by reusing the results of reductions of other integrals, we can lower the number of invocations still more: in the admittedly extreme case where we reduce all the above integrals in order 


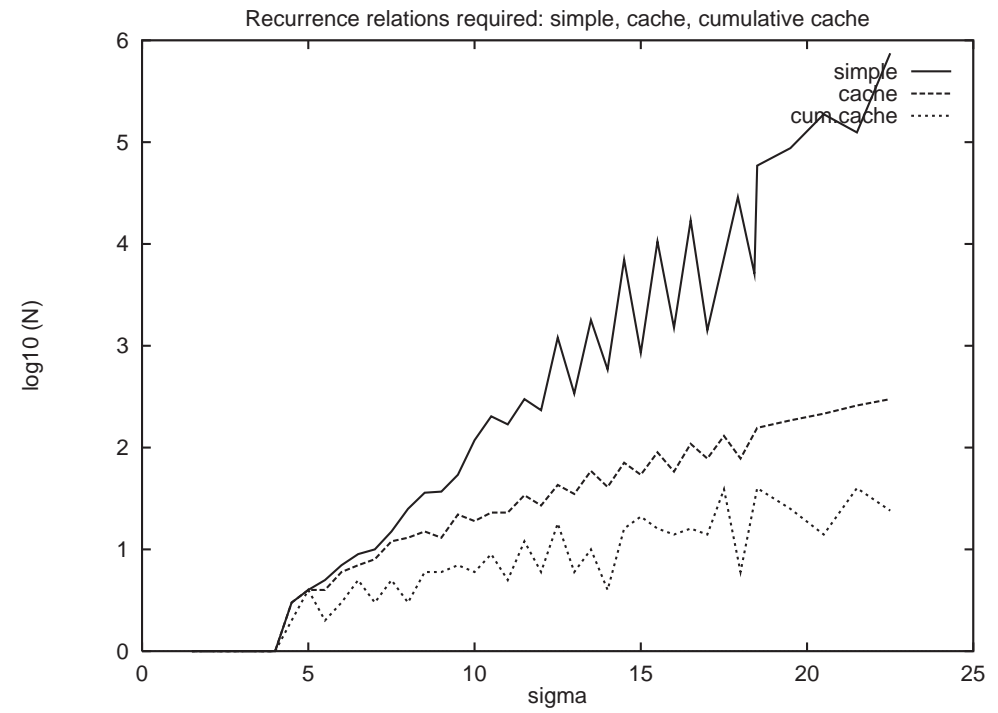

FiguRE 1. Maximum number of invocations of recurrence relations required to completely reduce integrals between $p=$ $[5,5,5,4,4]$ and $p=[-3,-3,-3,-4,-4]$, for the naïve algorithm, a variant which caches intermediate results for each reduction, and a further variant which cumulatively caches results. The logarithm of the number is plotted against the distance $\sigma$ from the target as defined in (77). The number of invocations required can be massively reduced by an implementation which caches results.

from low to high $\sigma$, we find the third curve of Figure 1, where successive high- $\sigma$ integrals need only tens of extra calls of the recurrence relations. The total number of recurrence relations employed in the three cases was 2244 836, 21234 and 2880 respectively.

\section{ACKNOWLDGMENTS}

I should particularly like to thank B. C. Carlson, who provided me with references Car98, [NP76] and, amongst other most helpful comments, pointed out an error in the original version of this paper. Though the error was relatively minor in itself, resolving it prompted me to thoroughly revise the algorithm, making it both more general and more symmetrical.

\section{REFERENCES}

[Car87] B. C. Carlson, A table of elliptic integrals of the second kind, Mathematics of Computation 49 (1987), no. 180, 595-606. MR 89b:65013

[Car88] B. C. Carlson, A table of elliptic integrals of the third kind, Mathematics of Computation 51 (1988), no. 183, 267-280. MR 89k:33003

[Car89] B. C. Carlson, A table of elliptic integrals: Cubic cases, Mathematics of Computation 53 (1989), no. 187, 327-333. MR 89m:65009

[Car91] B. C. Carlson, A table of elliptic integrals: One quadratic factor, Mathematics of Computation 56 (1991), no. 193, 267-280. MR 92b:33056

[Car98] B. C. Carlson, Elliptic integrals: Symmetry and symbolic integration, Atti dei Convengni Lincei 147 (1998), 161-181. MR 2001f:33024 
[Car99] B. C. Carlson, Toward symbolic integration of elliptic integrals, J. Symbolic Computation 28 (1999), no. 6, 739-753. MR 2001f:33025

[GR94] I. S. Gradshteyn and I. M. Ryzhik, Table of integrals, series, and products, 5th ed., Academic Press, 1994. MR 94g:00008

[NP76] Edward W. Ng and Desanka Polajnar, A study of alternative methods for the symbolic calculation of elliptic integrals, Proceedings of the 1976 ACM Symposium on Symbolic and Algebraic Computation, 1976, pp. 372-376.

Department of Physics and Astronomy, University of Glasgow, Glasgow, G12 8QQ,

UNITED KINGDOM

E-mail address: norman@astro.gla.ac.uk

$U R L$ : http://www.astro.gla.ac.uk/users/norman/ 